\title{
Article
}

\section{On Multivalent Analytic Functions Considered by a Multi-Arbitrary Differential Operator in a Complex Domain}

\author{
Najla M. Alarifi ${ }^{1, *,+(\mathbb{D}) \text { and Rabha W. Ibrahim }}{ }^{2,+}+\mathbb{C}$ \\ 1 Department of Mathematics, Imam Abdulrahman Bin Faisal University, Dammam 31113, Saudi Arabia \\ 2 Institute of Electrical and Electronics Engineers, Kuala Lumpur 59200, Malaysia; rabhaibrahim@yahoo.com \\ * Correspondence: nalareefi@iau.edu.sa \\ + These authors contributed equally to this work.
}

Citation: Alarif, N.M.; Ibrahim, R.W. On Multivalent Analytic Functions Considered by a Multi-Arbitrary Differential Operator in a Complex Domain. Axioms 2021, 10, 315. https://doi.org/10.3390/ axioms10040315

Academic Editor: Andriy Bandura

Received: 27 October 2021

Accepted: 15 November 2021

Published: 23 November 2021

Publisher's Note: MDPI stays neutral with regard to jurisdictional claims in published maps and institutional affiliations.

Copyright: (c) 2021 by the authors. Licensee MDPI, Basel, Switzerland. This article is an open access article distributed under the terms and conditions of the Creative Commons Attribution (CC BY) license (https:// creativecommons.org/licenses/by/ $4.0 /)$.

\begin{abstract}
Background: There is an increasing amount of information in complex domains, which necessitates the development of various kinds of operators, such as differential, integral, and linear convolution operators. Few investigations of the fractional differential and integral operators of a complex variable have been undertaken. (2) Methods: In this effort, we aim to present a generalization of a class of analytic functions based on a complex fractional differential operator. This class is defined by utilizing the subordination and superordination theory. (3) Results: We illustrate different fractional inequalities of starlike and convex formulas. Moreover, we discuss the main conditions to obtain sandwich inequalities involving the fractional operator. (4) Conclusion: We indicate that the suggested class is a generalization of recent works and can be applied to discuss the upper and lower bounds of a special case of fractional differential equations.
\end{abstract}

Keywords: univalent function; analytic function; subordination and superordination; open unit disk; special function; fractional calculus; fractional differential operator

\section{Introduction}

Geometric function theory's primary research objective is to introduce new classes of analytic functions and to explore their geometric shapes. There are many classes of analytic functions in the open unit disk, such as normalized, multivalent, harmonic and meromorphic functions, formulating different geometric processes. These processes present a derivative, integral or convolution operationally-for example, the Salagean differential operator [1] and its generalizations [2,3], conformabale differential operator [4] and symmetric differential operator [5]. Recently, fractional differential and integral operators have been utilized to formulate various types of generalizations of analytic functions. The most popular fractional operators are Riemann-Liouville fractional differential and integral operators. These operators were extended to the complex plane by Owa and Srivastava [6] and generalized for 2D-parametric fractional power in $[7,8]$.

The most significant embellishment of geometric classes is accomplished by the use of the subordination notion [9]. Such a presentation, for the first time, is given by Ma and Minda [10] for the class of normalized functions $f(0)=f^{\prime}(0)-1=0$. They have introduced the starlikeness and convexity sub-classes. These classes were modified later considering other collection of analytic functions, generalized by assuming any types of differential or integral operators and extended including fractional operators in the open unit disk (see [11-16]). In our investigation, we formulate the suggested class using the Ma-Minda-Janowski inequality. The descried classes are based on starlike and bounded turning functions.

In this study, we aim to present two new classes of multivalent analytic function types based on the generalized fractional differential operator. We shall show that the suggested fractional differential operator belongs to the same class of multivalent functions under a suitable process. Furthermore, by the Noshiro-Warschawski and Kaplan theorems, we 
show that the new operator is bounded turning in the open unit disk. Our method is based on the differential subordination theory. The suggested classes are of the starlike and bounded formula in the open unit disk. We present the sufficient and necessary conditions to be in these classes. As a special case, we study a Janowski formula geometrically, where this class indicates the extreme class of analytic functions. Moreover, we introduce different studies of these classes, including a set of differential inequalities. The conditions of a sandwich inequality are illustrated.

\section{Methods}

We proceed to illustrate some concepts which are requested for our study.

\subsection{Definitions}

- We consider a class of $\rho$-valent analytic functions denoted by $\Lambda_{\rho}, \rho \in \mathbb{N}$ and taking the series

$$
\varphi(z)=z^{\rho}+\sum_{n=\rho+1}^{\infty} \varphi_{n} z^{n}, z \in \sqcup:=\{z \in \mathbb{C},|z|<1\} .
$$

Two analytic functions $\varphi, \psi \in \Lambda_{\rho}$ are convoluted $(\varphi * \psi)$ if and only if

$$
\begin{gathered}
(\varphi * \psi)(z)=\left(z^{\rho}+\sum_{n=\rho+1}^{\infty} \varphi_{n} z^{n}\right) *\left(z^{\rho}+\sum_{n=\rho+1}^{\infty} \psi_{n} z^{n}\right) \\
=z^{\rho}+\sum_{n=\rho+1}^{\infty}\left(\varphi_{n} \psi_{n}\right) z^{n} .
\end{gathered}
$$

Clearly, when $\rho=1$, we have the normalized class of analytic functions in $\sqcup$.

- In addition, we introduce the class $\mathcal{P}$ of all analytic functions $\wp$ in $\sqcup$ with a positive real part in $\sqcup$ and $\wp(0)=1$. Associated with this class, we have the following sub-classes $\varsigma_{\rho}^{*}(\wp), \top_{\rho}^{v}(\wp)$, satisfying the inequality

$$
\frac{z \varphi^{\prime}(z)}{\rho \varphi(z)} \prec \wp(z), \quad z \in \sqcup
$$

and

$$
\left(\frac{\varphi(z)}{z^{\rho}}\right)^{v} \prec \wp(z), \quad z \in \sqcup
$$

accordingly, where $\prec$ indicates the subordination symbol [9].

- The extended Mittag-Leffler function is given by [17-19]

$$
\begin{gathered}
\Sigma_{a, b}^{p}(z)=\sum_{n=0}^{\infty} \frac{(p)_{n}}{\Gamma(b n+a)} \frac{z^{n}}{n !}, \\
\left((p)_{0}=1,(p)_{n}=p(p+1) \ldots(p+n-1)\right)
\end{gathered}
$$

where $(p)_{n}$ represents the Pochhammer symbol and

$$
\Sigma_{a, b}^{1}(z):=\Sigma_{a, b}(z)=\sum_{n=0}^{\infty} \frac{z^{n}}{\Gamma(b n+a)} .
$$

Note that $\Sigma_{a, b}^{p}(z)$ is the generalization of the function $e^{z}$, where $\Sigma_{1,1}^{1}(z)=e^{z}$. 


\section{2. $\rho$-Valent Fractional Differential Operator}

The Prabhakar fractional integral operator is formulated, for analytic function $f \in \sqcup$ by $[20,21]$

$$
\begin{aligned}
\prod_{a, b}^{p, q} f(z) & =\int_{0}^{z}(z-\zeta)^{b-1} \Sigma_{a, b}^{p}\left[q(z-\zeta)^{a}\right] f(\zeta) d \zeta \\
& =\left(f \cdot \varrho_{a, b}^{p, q}\right)(z), \quad(a, b, p, q \in \mathbb{C}, z \in \sqcup)
\end{aligned}
$$

where

$$
\left(\varrho_{a, b}^{p, q}\right)(z):=z^{b-1} \Sigma_{a, b}^{p}\left(q z^{a}\right)
$$

and

$$
\Sigma_{a, b}^{p}(z)=\sum_{n=0}^{\infty} \frac{\Gamma(p+n)}{\Gamma(p) \Gamma(a n+b)}\left(\frac{z^{n}}{n !}\right) .
$$

For instant, for $\psi(z)=z^{\ell-1}$, we obtain (see [22]—Corollary 2.3)

$$
\begin{aligned}
\prod_{a, b}^{p, q} z^{\ell-1} & =\int_{0}^{z}(z-\zeta)^{b-1} \Sigma_{a, b}^{p}\left[q(z-\zeta)^{a}\right]\left(\zeta^{\ell-1}\right) d \zeta \\
& =\Gamma(\ell) z^{b+\ell-1} \Sigma_{a, b+\ell}^{p}\left(q z^{a}\right) .
\end{aligned}
$$

Corresponding to the integral $\prod_{a, b}^{p, q}$, the differential construction is formulated by [20]

$$
{ }_{\kappa} \Delta_{a, b}^{p, q} f(z)=\frac{d^{\kappa}}{d z^{\kappa}}\left(\prod_{a, \kappa-b}^{-p, q} f(z)\right), \quad \kappa \in \mathbb{N} .
$$

Definition 1. Let $\varphi \in \Lambda_{\rho}$. Then, the $\rho$-valent differential operator of (2) can be realized in view of the Riemann-Liouville derivative by:

$$
\begin{aligned}
& { }_{\kappa}^{\mathcal{R}} \Delta_{a, b}^{p, q} \varphi(z)=\frac{d^{\kappa}}{d z^{\kappa}} \int_{0}^{z}(z-\zeta)^{\kappa-b-1} \Sigma_{a, \kappa-b}^{-p}\left[q(z-\zeta)^{\alpha}\right] \varphi(\zeta) d \zeta \\
& =\frac{d^{\kappa}}{d z^{\kappa}}\left(\prod_{a, \kappa-b}^{-p, q} \varphi(z)\right) .
\end{aligned}
$$

The $\rho$-valent differential operator in the structure of the Caputo derivative can be recognized coordinately by

$$
\begin{gathered}
{ }_{\kappa}^{\mathcal{C}} \Delta_{a, b}^{p, q} \varphi(z)=\int_{0}^{z}(z-\zeta)^{\kappa-b-1} \Sigma_{a, \kappa-b}^{-p}\left[q(z-\zeta)^{a}\right]\left(\frac{d^{\kappa}}{d \zeta^{\kappa}} \varphi(\zeta)\right) d \zeta . \\
=\prod_{a, \kappa-b}^{-p, q}\left(\frac{d^{\kappa}}{d z^{\kappa}} \varphi(z)\right) .
\end{gathered}
$$

Note that

$$
{ }_{\kappa}^{\mathcal{C}} \Delta_{a, b}^{p, q} \varphi(z)={ }_{\kappa}^{\mathcal{R}} \Delta_{a, b}^{p, q} \varphi(z)-\sum_{j=0}^{\kappa-1} z^{j-b} \Sigma_{a, j-b}^{-p}\left[\omega z^{a}\right] \varphi^{(j)}(0) .
$$

Since $\varphi^{(j)}(0)=0, j=\rho-1$, then we have

$$
{ }_{\kappa}^{\mathcal{C}} \Delta_{a, b}^{p, q} \varphi(z)={ }_{\kappa}^{\mathcal{R}} \Delta_{a, b}^{p, q} \varphi(z) .
$$


For instant, suppose that $\varphi(z)=z^{\rho}, \rho \geq 1$, ref. [22], Corollary 2.3, implies that

$$
\begin{aligned}
{ }_{1}^{C} \Delta_{a, b}^{p, q}\left(z^{\rho}\right) & =\int_{0}^{z}(z-\zeta)^{1-b-1} \Sigma_{a, 1-b}^{-p}\left[q(z-\zeta)^{a}\right]\left(\frac{d}{d \zeta} \varphi(\zeta)\right) d \zeta \\
& =\int_{0}^{z}(z-\zeta)^{s-1} \Sigma_{a, s}^{-p}\left[q(z-\zeta)^{a}\right]\left(\frac{d}{d \zeta}\left(\zeta^{\rho}\right)\right) d \zeta \\
& =\rho \int_{0}^{z} \zeta^{\rho-1}(z-\zeta)^{s-1} \Sigma_{a, s}^{-p}\left[q(z-\zeta)^{a}\right] d \zeta \\
& =\Gamma(\rho+1) z^{s+\rho-1} \Sigma_{a, s+\rho}^{-p}\left[q z^{\alpha}\right], \quad s:=1-b .
\end{aligned}
$$

In general, we have

$$
\begin{aligned}
{ }_{\kappa}^{C} \Delta_{a, b}^{p, q}\left(z^{\rho}\right) & =\int_{0}^{z}(z-\zeta)^{\kappa-b-1} \Sigma_{a, \kappa-b}^{-p}\left[q(z-\zeta)^{a}\right]\left(\frac{d^{\kappa}}{d \zeta^{\kappa}}\left(\zeta^{\rho}\right)\right) d \zeta \\
& =(1-\kappa+\rho)_{\mathcal{\kappa}} \int_{0}^{z} \zeta^{\rho-\kappa}(z-\zeta)^{\kappa-b-1} \Sigma_{a, \kappa-b}^{-p}\left[q(z-\zeta)^{a}\right] d \zeta \\
& =(1-\kappa+\rho)_{\mathcal{\kappa}} \int_{0}^{z} \zeta^{(\rho-\kappa+1)-1}(z-\zeta)^{\kappa-b-1} \Sigma_{a, \kappa-b}^{-p}\left[q(z-\zeta)^{a}\right] d \zeta \\
& :=(t)_{\mathcal{\kappa}} \int_{0}^{z} \zeta^{t-1}(z-\zeta)^{s-1} \Sigma_{a, s}^{-p}\left[q(z-\zeta)^{a}\right] d \zeta \\
& =(t)_{\mathcal{K}} \Gamma(t) z^{t+s-1} \Sigma_{a, s+t}^{-p}\left[q z^{a}\right],
\end{aligned}
$$

where $s:=\kappa-b, t:=\rho-\kappa+1$ and $(t)_{\kappa}=\frac{\Gamma(1+\rho)}{\Gamma(1+\rho-\kappa)}$. Consequently, we obtain

$$
\begin{aligned}
{ }_{\kappa}^{\mathcal{C}} \Delta_{a, b}^{p, q}\left(z^{\rho}\right) & =\Gamma(1+\rho) z^{s+t-1} \Sigma_{a, s+t}^{-p}\left[q z^{a}\right] \\
& =\Gamma(\kappa+t) z^{t+s-1} \Sigma_{a, s+t}^{-p}\left[q z^{a}\right] .
\end{aligned}
$$

As a conclusion, we realize the next property

Proposition 1. Let $\varphi \in \Lambda_{\rho}$. Define the operator ${ }_{\kappa}^{\mathcal{C}} \Omega_{a, b}^{p, q}: \sqcup \rightarrow \sqcup$ by

$$
{ }_{\kappa}^{c} \Omega_{a, b}^{p, q}:=\left(\frac{z^{\rho}}{(t)_{\mathcal{K}} \Gamma(t) z^{t+s-1} \Sigma_{a, s+t}^{-p}\left[q z^{a}\right]}\right)\left({ }_{\kappa}^{c} \Delta_{a, b}^{p, q}\right) .
$$

Then ${ }_{\kappa}^{\mathcal{C}} \Omega_{a, b}^{p, q} \varphi \in \Lambda_{\rho}$.

$$
\left(a, b, p, q \in \mathbb{C}, z \in \sqcup, \varphi \in \Lambda_{\rho}\right)
$$

and

$$
(s:=\kappa-b, \quad t:=\rho-\kappa+1, \quad \tau:=n-\kappa+1) .
$$

Proof. Assume that $\varphi \in \Lambda_{\rho}$. Then, a calculation yields 


$$
\begin{aligned}
{ }_{\kappa}^{\mathcal{C}} \Omega_{a, b}^{p, q} \varphi(z)= & \left(\frac{z^{\rho}}{(t)_{\mathcal{K}} \Gamma(t) z^{t+s-1} \Sigma_{a, s+t}^{-p}\left[q z^{a}\right]}\right){ }_{\kappa}^{\mathcal{C}} \Delta_{a, b}^{p, q} \varphi(z) \\
& =\left(\frac{z^{\rho}}{(t)_{\mathcal{K}} \Gamma(t) z^{t+s-1} \Sigma_{a, s+t}^{-p}\left[q z^{a}\right]}\right){ }_{\kappa}^{\mathcal{C}} \Delta_{a, b}^{p, q}\left(z^{\rho}+\sum_{n=\rho+1}^{\infty} \varphi_{n} z^{n}\right) \\
& =\left(\frac{z^{\rho}}{(t)_{\mathcal{K}} \Gamma(t) z^{t+s-1} \Sigma_{a, s+t}^{-p}\left[q z^{a}\right]}\right)\left({ }_{\kappa}^{\mathcal{C}} \Delta_{a, b}^{p, q} z^{\rho}+\sum_{n=\rho+1}^{\infty} \varphi_{n}{ }_{\mathcal{K}}^{\mathcal{C}} \Delta_{a, b}^{p, q} z^{n}\right) \\
& =\left(\frac{z^{\rho}}{(t)_{\mathcal{K}} \Gamma(t) z^{t+s-1} \Sigma_{a, s+t}^{-p}\left[q z^{a}\right]}\right)\left((t)_{\mathcal{K}} \Gamma(t) z^{t+s-1} \Sigma_{a, s+t}^{-p}\left[q z^{a}\right]+\sum_{n=\rho+1}^{\infty} \varphi_{n}{ }_{\kappa}^{\mathcal{C}} \Delta_{a, b}^{p, q} z^{n}\right) \\
& =z^{\rho}+\left(\frac{z^{\rho}}{(t)_{\mathcal{K}} \Gamma(t) z^{t+s-1} \Sigma_{a, s+t}^{-p}\left[q z^{a}\right]}\right) \sum_{n=\rho+1}^{\infty} \varphi_{n}(\tau)_{\mathcal{K}} \Gamma(\tau) z^{\tau+s-1} \Sigma_{a, s+\tau}^{-p}\left[q z^{a}\right] \\
& =z^{\rho}+\sum_{n=\rho+1}^{\infty}\left(\frac{(\tau)_{\mathcal{K}} \Gamma(\tau) \Sigma_{a, s+\tau}^{-p}\left[q z^{a}\right]}{(t)_{\mathcal{K}} \Gamma(t) \Sigma_{a, s+t}^{-p}\left[q z^{a}\right]}\right) \varphi_{n} z^{n} \\
& =z^{\rho}+\sum_{n=\rho+1}^{\infty} \omega_{n} \varphi_{n} z^{n}
\end{aligned}
$$

where

$$
\omega_{n}:=\left(\frac{(n-\kappa+1)_{\kappa} \Gamma(n-\kappa+1) \Sigma_{a, 1-b+n}^{-p}\left[q z^{a}\right]}{(\rho-\kappa+1)_{\kappa} \Gamma(\rho-\kappa+1) \Sigma_{a, \rho+1-b}^{-p}\left[q z^{a}\right]}\right) .
$$

This yields ${ }_{\kappa}^{\mathcal{C}} \Omega_{a, b}^{p, q} \varphi \in \Lambda_{\rho}$. Moreover,

$$
{ }_{\kappa}^{\mathcal{C}} \Omega_{a, b}^{p, q} \varphi(z)={ }_{\kappa}^{\mathcal{C}} \Omega_{a, b}^{p, q}(z) * \varphi(z) .
$$

We call ${ }_{\kappa}^{\mathcal{C}} \Omega_{a, b}^{p, q}$, the $\rho$-valent Prabhakar operator in the open unit disk. Since ${ }_{\kappa}^{\mathcal{C}} \Omega_{a, b}^{p, q} \in$ $\Lambda_{\rho}$, then we can investigate its properties in the recommendation of the geometric function theory. Our goal is to formulate it in terms of some well known sub-classes of analytic functions. It is clear that $\omega_{n}$ is a complex connection (coefficient) of the operator and it is a constant when $a=0$. In addition, we have

$$
\lim _{\rho \rightarrow n} \omega_{n}=\left(\frac{(n-\kappa+1)_{\kappa} \Gamma(n-\kappa+1) \Sigma_{a, 1-b+n}^{-p}\left[q z^{a}\right]}{(n-\kappa+1)_{\kappa} \Gamma(n-\kappa+1) \Sigma_{a, 1-b+n-}^{-p}\left[q z^{a}\right]}\right)=1 .
$$

Remark 1. The integral operator corresponds to the fractional differential operator ${ }_{k}^{\mathcal{C}} \Delta_{\alpha, \beta}^{\gamma, \omega}$, which is expanded by the series

$$
{ }_{\kappa}^{\mathcal{C}} \Xi_{a, b}^{p, q} \varphi(z)=z^{\rho}+\sum_{n=\rho+1}^{\infty}\left(\frac{(t)_{\kappa} \Gamma(t) \Sigma_{a, s+t}^{-p}\left[q z^{a}\right]}{(\tau)_{\kappa} \Gamma(\tau) \Sigma_{a, s+\tau}^{-p}\left[q z^{a}\right]}\right) \varphi_{n} z^{n} .
$$

It is clear that

$$
\left({ }_{\kappa}^{\mathcal{C}} \Omega_{a, b}^{p, q} *{ }_{\kappa}^{\mathcal{C}} \Xi_{a, b}^{p, q}\right) \varphi(z)=\left({ }_{\kappa}^{\mathcal{C}} \Xi_{a, b}^{p, q} *{ }_{\kappa}^{\mathcal{C}} \Omega_{a, b}^{p, q}\right) \varphi(z)=\varphi(z) .
$$

The linear convex combination of the operators ${ }_{\kappa}^{C} \Omega_{a, b}^{p, q} \varphi(z)$ and ${ }_{\kappa}^{\mathcal{C}} \Xi_{a, b}^{p, q} \varphi(z)$ can be formulated as follows:

$$
{ }_{\kappa}^{\alpha} \partial_{a, b}^{p, q} \varphi(z)=\alpha{ }_{\kappa}^{\mathcal{C}} \Omega_{a, b}^{p, q} \varphi(z)+(1-\alpha){ }_{\kappa}^{\mathcal{C}} \Xi_{a, b}^{p, q} \varphi(z),
$$


where $\alpha \in[0,1]$. Certainly, ${ }_{k}^{\alpha} \mathrm{\partial}_{a, b}^{p, q} \varphi \in \Lambda_{\rho}$, where $\varphi \in \Lambda_{\rho}$.

\subsection{Generalized Subordination Formulas}

By utilizing the $\rho$-valent differential operator ${ }_{\kappa}^{\mathcal{C}} \Omega_{a, b}^{p, q} \varphi$, we can obtain the generalized sub-classes of the classes

$$
\frac{z \varphi^{\prime}(z)}{\rho \varphi(z)} \prec \wp(z), \quad z \in \sqcup
$$

and

$$
\left(\frac{\varphi(z)}{z^{\rho}}\right)^{v} \prec \wp(z), \quad z \in \sqcup
$$

as follows:

Definition 2. A function $\varphi \in \Lambda_{\rho}$ is in the class ${ }_{\kappa}^{\mathcal{C}} \varsigma_{a, b}^{* p, q}(\wp)$ if and only if

$$
{ }_{\kappa}^{\mathcal{C}} \varsigma_{\alpha, \beta}^{* \gamma, \omega}(\wp)=\left\{\varphi \in \Lambda_{\rho}: \frac{z\left({ }_{\kappa}^{\mathcal{C}} \Omega_{a, b}^{p, q} \varphi(z)\right)^{\prime}}{\rho_{\mathcal{K}}^{\mathcal{C}} \Omega_{a, b}^{p, q} \varphi(z)} \prec \wp(z), \wp(0)=1\right\} .
$$

And the sub-class

Definition 3. A function $\varphi \in \Lambda_{\rho}$ is in the class ${ }_{\kappa}^{v} \top_{a, b}^{p, q}(\wp)$ if and only if

$$
{ }_{\kappa}^{v} \top_{a, b}^{p, q}(\wp)=\left\{\varphi \in \Lambda_{\rho}:\left(\frac{{ }_{\kappa}^{\mathcal{C}} \Omega_{a, b}^{p, q} \varphi(z)}{z^{\rho}}\right)^{v} \prec \wp(z), \wp(0)=1\right\} .
$$

We aim to study the above classes in view of geometric function theory. We conclude some facts and connections between these two classes.

The following preliminaries are requested to prove our results

Lemma 1. (See [9].) Consider the general class of holomorphic functions, as follows:

$$
\mathbb{O}[h, n]=\left\{\hbar: \hbar(z)=h+h_{n} z^{n}+h_{n+1} z^{n+1}+\ldots\right\} .
$$

Assume that:

a. $u \in \mathbb{R}$. Then, $\Re\left(\hbar(z)+u z \hbar^{\prime}(z)\right)>0 \rightarrow \Re(\hbar(z))>0$. Furthermore, suppose that $u>0$ and $\hbar \in \mathbb{O}[1, n]$. Then, for the constants $u_{1}>0$ and $u_{2}>0$ with $u_{2}=u_{2}\left(u, u_{1}, n\right)$,

$$
\hbar(z)+u z \hbar^{\prime}(z) \prec\left(\frac{1+z}{1-z}\right)^{u_{2}} \rightarrow \hbar(z) \prec\left(\frac{1+z}{1-z}\right)^{u_{1}} .
$$

b. $v \in[0,1)$ and $\hbar \in \mathbb{O}[1, n]$ and a positive constant $w>0$. Then, the real formula is $\Re\left(\hbar^{2}(z)+2 \hbar(z) \cdot z \hbar^{\prime}(z)\right)>v \rightarrow \Re(\hbar(z))>w$.

c. $\hbar \in \mathbb{O}[h, n]$. Then, $\Re\left(\hbar(z)+z \hbar^{\prime}(z)+z^{2} \hbar^{\prime \prime}(z)\right)>0 \rightarrow \Re(\hbar)>0$. Additionally, let $\jmath: \sqcup \rightarrow \mathbb{R}$. Then,

$$
\Re\left(\hbar(z)+\jmath(z) \frac{z \hbar^{\prime}(z)}{\hbar(z)}\right)>0 \rightarrow \Re(\hbar(z))>0 .
$$

d. $\hbar, \wp \in \mathbb{O}[h, n]$, where $\wp$ is convex univalent in $\sqcup$ and for $v_{1}, v_{2} \in \mathbb{C}, v_{2} \neq 0, v_{1} \hbar(z)+$ $v_{2} z \hbar^{\prime}(z) \prec v_{1} \wp(z)+v_{2} z \wp^{\prime}(z) \rightarrow \hbar(z) \prec \wp(z)$. (See [23].)

e. $\hbar, \wp \in \mathbb{O}[h, n]$, where $\wp$ is convex univalent in $\sqcup$ such that $\hbar(z)+v z \hbar^{\prime}(z)$ is univalent; then, $\wp(z)+v z \wp^{\prime}(z) \prec \hbar(z)+v z \hbar^{\prime}(z) \rightarrow \wp(z) \prec \hbar(z)$. (See [24].)

f. $\quad \hbar, \hbar, g \in \mathbb{O}[h, n]$ and $g$ is convex univalent in $\sqcup$ such that $\hbar \prec g$ and $\hbar \prec g$; then, $\lambda \hbar+(1-$ $\lambda) \hbar \prec g, \lambda \in[0,1]$. (See [25].) 
g. $\quad \hbar, g \in \mathbb{O}[h, n]$ and $g$ is convex univalent in $\sqcup$ such that $\hbar(z)+(1 / \ell)\left(z \hbar^{\prime}(z)\right) \prec g(z), \ell \neq 0$; then (see [9]-Theorem 3.1b, P71),

$$
\hbar(z) \prec\left(\frac{\ell}{n}\right) z^{-(\ell / n)} \int_{0}^{z}\left(g(\xi) \xi^{\frac{\ell}{n}-1}\right) d \xi \prec g(z), \quad z \in \sqcup .
$$

h. $\quad \hbar, g \in \mathbb{O}[1, n]$ such that

$$
\hbar(z)=1+\sum_{n=1}^{\infty} h_{n} z^{n}, \quad g=1+\sum_{n=1}^{\infty} g_{n} z^{n}
$$

and $\hbar \prec g$ then for all $n,\left|h_{n}\right| \leq\left|g_{1}\right|$. (See [26].)

\section{Results}

We first show that $\left(\frac{{ }_{\kappa}^{\mathcal{C}} \Omega_{a, b}^{p, q} \varphi(z)}{z^{\rho}}\right)^{v}$ is a positive real part.

Theorem 1. Assume that one of the following relations occurs

- $\Re\left\{\frac{z\left({ }_{\kappa}^{\mathcal{C}} \Omega_{a, b}^{p, q} \varphi(z)\right)^{\prime}+(1-\rho)\left({ }_{\kappa}^{\mathcal{C}} \Omega_{a, b}^{p, q} \varphi(z)\right)}{z^{\rho}}\right\}>0$;

- $\frac{z\left({ }_{\kappa}^{\mathcal{C}} \Omega_{a, b}^{p, q} \varphi(z)\right)^{\prime}+(1-\rho)\left({ }_{\kappa}^{\mathcal{C}} \Omega_{a, b}^{p, q} \varphi(z)\right)}{z^{\rho}} \prec\left(\frac{1+z}{1-z}\right)^{u_{2}}, \quad u_{2}>0, z \in \sqcup ;$

- $\quad \Re\left(\frac{\left({ }_{\kappa}^{\mathcal{C}} \Omega_{a, b}^{p, q} \varphi(z)\right)}{z^{2 \rho}}\left(2 z\left({ }_{\kappa}^{\mathcal{C}} \Omega_{a, b}^{p, q} \varphi(z)\right)^{\prime}+(1-2 \rho)\left({ }_{\kappa}^{\mathcal{C}} \Omega_{a, b}^{p, q} \varphi(z)\right)\right)\right)>\varepsilon, \varepsilon \in[0,1)$;

- $\quad \Re\left(\frac{z^{2}\left({ }_{\kappa}^{\mathcal{C}} \Omega_{a, b}^{p, q} \varphi(z)\right)^{\prime \prime}+(1-2 \rho) z\left({ }_{\kappa}^{\mathcal{C}} \Omega_{a, b}^{p, q} \varphi(z)\right)^{\prime}+\left(\rho^{2}+1\right)\left({ }_{\kappa}^{\mathcal{C}} \Omega_{a, b}^{p, q} \varphi(z)\right)}{z^{\rho}}\right)>0$;

- $\quad \Re\left(\frac{z\left({ }_{\kappa}^{\mathcal{C}} \Omega_{a, b}^{p, q} \varphi(z)\right)^{\prime}}{\left({ }_{\mathcal{K}}^{\mathcal{C}} \Omega_{a, b}^{p, q} \varphi(z)\right)}+\frac{\left({ }_{\kappa}^{\mathcal{C}} \Omega_{a, b}^{p, q} \varphi(z)\right)}{z^{\rho}}\right)>\rho ;$

then, $\frac{\left({ }_{\kappa}^{\mathcal{C}} \Omega_{a, b}^{p, q} \varphi(z)\right)}{z^{\rho}} \in \mathcal{P}(\varepsilon)$ for some $\varepsilon \in[0,1)$.

Proof. Singe $v$ as follows:

$$
v(z)=\frac{\left({ }_{\kappa}^{\mathcal{C}} \Omega_{a, b}^{p, q} \varphi(z)\right)}{z^{\rho}}, \quad z \in \sqcup .
$$

Then, a calculation yields

$$
\begin{aligned}
z v^{\prime}(z)+v(z) & =\frac{z\left({ }_{\mathcal{K}}^{\mathcal{C}} \Omega_{a, b}^{p, q} \varphi(z)\right)^{\prime}-\rho\left({ }_{\kappa}^{\mathcal{C}} \Omega_{a, b}^{p, q} \varphi(z)\right)}{z^{\rho}}+\frac{\left({ }_{\mathcal{K}}^{\mathcal{C}} \Omega_{a, b}^{p, q} \varphi(z)\right)}{z^{\rho}} \\
& =\frac{z\left({ }_{\kappa}^{\mathcal{C}} \Omega_{a, b}^{p, q} \varphi(z)\right)^{\prime}+(1-\rho)\left({ }_{\kappa}^{\mathcal{C}} \Omega_{a, b}^{p, q} \varphi(z)\right)}{z^{\rho}}
\end{aligned}
$$

Consequently, we obtain $\Re\left(z v^{\prime}(z)+v(z)\right)>0$. Thus, Lemma 1 (a-first part), with $u=1$ implies that $\Re(v(z))>0$, which means that $v(z) \in \mathcal{P}(\varepsilon)$. Consequently, the second part of the theorem comes from Lemma 1 (a-second part)

$$
v(z) \prec\left(\frac{1+z}{1-z}\right)^{u_{1}}
$$




\section{A computation gives}

$$
\begin{aligned}
& \Re\left(v^{2}(z)+2 v(z) \cdot z v^{\prime}(z)\right) \\
& =\Re\left(\left(\frac{\left({ }_{\kappa}^{\mathcal{C}} \Omega_{a, b}^{p, q} \varphi(z)\right)}{z^{\rho}}\right)^{2}+2\left(\frac{\left({ }_{\kappa}^{\mathcal{C}} \Omega_{a, b}^{p, q} \varphi(z)\right)}{z^{\rho}}\right)\left(z\left(\frac{\left({ }_{\kappa}^{\mathcal{C}} \Omega_{a, b}^{p, q} \varphi(z)\right)}{z^{\rho}}\right)\right)\right) \\
& =\Re\left(\left(\frac{\left({ }_{k}^{\mathcal{C}} \Omega_{a, b}^{p, q} \varphi(z)\right)}{z^{\rho}}\right)^{2}+2\left(\frac{\left({ }_{k}^{\mathcal{C}} \Omega_{a, b}^{p, q} \varphi(z)\right)}{z^{\rho}}\right)\left(\frac{z\left({ }_{k}^{\mathcal{C}} \Omega_{a, b}^{p, q} \varphi(z)\right)^{\prime}-\rho\left({ }_{k}^{\mathcal{C}} \Omega_{a, b}^{p, q} \varphi(z)\right)}{z^{\rho}}\right)\right) \\
& =\Re\left(\frac{\left({ }_{\kappa}^{\mathcal{C}} \Omega_{a, b}^{p, q} \varphi(z)\right)}{z^{2 \rho}}\left(2 z\left({ }_{\kappa}^{\mathcal{C}} \Omega_{a, b}^{p, q} \varphi(z)\right)^{\prime}-2 \rho\left({ }_{\kappa}^{\mathcal{C}} \Omega_{a, b}^{p, q} \varphi(z)\right)+\left({ }_{\kappa}^{\mathcal{C}} \Omega_{a, b}^{p, q} \varphi(z)\right)\right)\right) \\
& =\Re\left(\frac{\left({ }_{\kappa}^{\mathcal{C}} \Omega_{a, b}^{p, q} \varphi(z)\right)}{z^{2 \rho}}\left(2 z\left({ }_{\kappa}^{\mathcal{C}} \Omega_{a, b}^{p, q} \varphi(z)\right)^{\prime}+(1-2 \rho)\left({ }_{\kappa}^{\mathcal{C}} \Omega_{a, b}^{p, q} \varphi(z)\right)\right)\right) \\
& >\varepsilon \text {. }
\end{aligned}
$$

In view of Lemma $1 \mathrm{~b}$, we have $v(z) \in \mathcal{P}(\varepsilon)$. Accordingly, the Noshiro-Warschawski and Kaplan theorems imply that $\left({ }_{\kappa}^{C} \Omega_{a, b}^{p, q} \varphi(z)\right)$ is a bounded turning function (univalent) in $\sqcup$.

Computing the real

$$
\begin{aligned}
& \Re\left(v(z)+z v^{\prime}(z)+z^{2} v^{\prime \prime}(z)\right) \\
& =\Re\left(\frac{\left({ }_{\kappa}^{\mathcal{C}} \Omega_{a, b}^{p, q} \varphi(z)\right)}{z^{\rho}}+z\left(\frac{\left({ }_{\kappa}^{c} \Omega_{a, b}^{p, q} \varphi(z)\right)}{z^{\rho}}\right)^{\prime}+z^{2}\left(\frac{\left({ }_{\kappa}^{c} \Omega_{a, b}^{p, q} \varphi(z)\right)}{z^{\rho}}\right)^{\prime \prime}\right) \\
& =\Re\left(\frac{\left({ }_{\kappa}^{\mathcal{C}} \Omega_{a, b}^{p, q} \varphi(z)\right)}{z^{\rho}}+\left(\frac{z\left({ }_{\kappa}^{\mathcal{C}} \Omega_{a, b}^{p, q} \varphi(z)\right)^{\prime}-\rho\left({ }_{\kappa}^{\mathcal{C}} \Omega_{a, b}^{p, q} \varphi(z)\right)}{z^{\rho}}\right)\right) \\
& +\Re\left(\frac{z^{2}\left({ }_{\kappa}^{C} \Omega_{a, b}^{p, q} \varphi(z)\right)^{\prime \prime}-2 \rho z\left({ }_{\kappa}^{C} \Omega_{a, b}^{p, q} \varphi(z)\right)^{\prime}+\rho^{2}\left({ }_{\kappa}^{C} \Omega_{a, b}^{p, q} \varphi(z)\right)+\rho\left({ }_{\kappa}^{C} \Omega_{a, b}^{p, q} \varphi(z)\right)}{z^{\rho}}\right) \\
& =\Re\left(\frac{z^{2}\left({ }_{\kappa}^{\mathcal{C}} \Omega_{a, b}^{p, q} \varphi(z)\right)^{\prime \prime}+(1-2 \rho) z\left({ }_{\kappa}^{\mathcal{C}} \Omega_{a, b}^{p, q} \varphi(z)\right)^{\prime}+\left(\rho^{2}+1\right)\left({ }_{\kappa}^{C} \Omega_{a, b}^{p, q} \varphi(z)\right)}{z^{\rho}}\right)
\end{aligned}
$$

$>0$. 
Similarly, we obtain

$$
\begin{aligned}
& \Re\left(v(z)+z \frac{v^{\prime}(z)}{v(z)}\right) \\
& =\Re\left(\frac{\left({ }_{\kappa}^{\mathcal{C}} \Omega_{a, b}^{p, q} \varphi(z)\right)}{z^{\rho}}+z \frac{\left(\frac{\left({ }_{\mathcal{K}}^{\mathcal{C}} \Omega_{a, b}^{p, q} \varphi(z)\right)}{z^{\rho}}\right)^{\prime}}{\frac{\left({ }_{\kappa}^{\mathcal{C}} \Omega_{a, b}^{p, q} \varphi(z)\right)}{z^{\rho}}}\right) \\
& =\Re\left(\frac{\left({ }_{\kappa}^{\mathcal{C}} \Omega_{a, b}^{p, q} \varphi(z)\right)}{z^{\rho}}+\frac{z\left({ }_{\kappa}^{\mathcal{C}} \Omega_{a, b}^{p, q} \varphi(z)\right)^{\prime}-\rho\left({ }_{\kappa}^{\mathcal{C}} \Omega_{a, b}^{p, q} \varphi(z)\right)}{\left({ }_{\kappa}^{\mathcal{C}} \Omega_{a, b}^{p, q} \varphi(z)\right)}\right) \\
& =\Re\left(\frac{\left({ }_{\kappa}^{\mathcal{C}} \Omega_{a, b}^{p, q} \varphi(z)\right)}{z^{\rho}}+\frac{z\left({ }_{\kappa}^{\mathcal{C}} \Omega_{a, b}^{p, q} \varphi(z)\right)^{\prime}}{\left({ }_{\kappa}^{\mathcal{C}} \Omega_{a, b}^{p, q} \varphi(z)\right)}-\rho\right) \\
& =\Re\left(\frac{z\left({ }_{\kappa}^{\mathcal{C}} \Omega_{a, b}^{p, q} \varphi(z)\right)^{\prime}}{\left({ }_{\mathcal{K}}^{\mathcal{C}} \Omega_{a, b}^{p, q} \varphi(z)\right)}+\frac{\left({ }_{\kappa}^{\mathcal{C}} \Omega_{a, b}^{p, q} \varphi(z)\right)}{z^{\rho}}\right)-\rho>0
\end{aligned}
$$

which is indicated by Lemma 1c $v \in \mathcal{P}(\varepsilon)$.

Corollary 1. Let the assumptions of Theorem 1 hold. Then, for a positive real number $v$,

$$
\Re\left(\frac{{ }_{\kappa}^{\mathcal{C}} \Omega_{a, b}^{p, q} \varphi(z)}{z^{\rho}}\right)^{v}>0, \quad z \in \sqcup .
$$

The next outcome confirms the optimistically of the functional $\left(\frac{{ }_{\kappa}^{\mathcal{C}} \Omega_{a, b}^{p, q} \varphi(z)}{z^{\rho}}\right)^{v}$

Theorem 2. Let $\varphi \in{ }_{\mathcal{K}}^{\mathcal{C}} S_{a, b}^{* p, q}(\wp)$ such that $\wp$ is a univalent convex function in $\sqcup$. Then, $\varphi \in$ ${ }_{\kappa}^{v} \top_{a, b}^{p, q}(\wp)$ for some $v \in(0,1]$.

Proof. Let $\varphi \in{ }_{\kappa}^{\mathcal{C}} S_{a, b}^{* p, q}(\wp)$ and

$$
V(z)=\left(\frac{{ }_{\kappa}^{\mathcal{C}} \Omega_{a, b}^{p, q} \varphi(z)}{z^{\rho}}\right)^{v}
$$

Taking in account that $v$ is an arbitrary positive real number, one can assume that $v=1 / \rho$; thus, a calculation indicates the following fact

$$
\begin{aligned}
V(z)+z V^{\prime}(z) & =\left(\frac{{ }_{\kappa}^{\mathcal{C}} \Omega_{a, b}^{p, q} \varphi(z)}{z^{\rho}}\right)^{v}+z\left(\left(\frac{{ }_{\kappa}^{\mathcal{C}} \Omega_{a, b}^{p, q} \varphi(z)}{z^{\rho}}\right)^{v}\right)^{\prime} \\
& =\left(\frac{{ }_{\kappa}^{\mathcal{C}} \Omega_{a, b}^{p, q} \varphi(z)}{z^{\rho}}\right)^{v}\left(v \frac{z\left({ }_{\kappa}^{\mathcal{C}} \Omega_{a, b}^{p, q} \varphi(z)\right)^{\prime}}{{ }_{\mathcal{K}}^{\mathcal{C}} \Omega_{a, b}^{p, q} \varphi(z)}+(1-v \rho)\right)
\end{aligned}
$$


which leads to

$$
\begin{aligned}
1+\frac{z V^{\prime}(z)}{V(z)} & =\left(v \frac{z\left({ }_{\mathcal{K}}^{\mathcal{C}} \Omega_{a, b}^{p, q} \varphi(z)\right)^{\prime}}{{ }_{\mathcal{K}}^{\mathcal{C}} \Omega_{a, b}^{p, q} \varphi(z)}+(1-v \rho)\right) \\
& =\frac{1}{\rho}\left(\frac{z\left({ }_{\mathcal{K}}^{\mathcal{C}} \Omega_{a, b}^{p, q} \varphi(z)\right)^{\prime}}{{ }_{\mathcal{K}}^{\mathcal{C}} \Omega_{a, b}^{p, q} \varphi(z)}\right) .
\end{aligned}
$$

Since $\varphi \in{ }_{\kappa}^{\mathcal{C}} S_{a, b}^{* p, q}(\wp)$,

$$
\left(\frac{z\left({ }_{\kappa}^{\mathcal{C}} \Omega_{a, b}^{p, q} \varphi(z)\right)^{\prime}}{\rho_{\mathcal{K}}^{\mathcal{C}} \Omega_{a, b}^{p, q} \varphi(z)}\right) \prec \wp(z) .
$$

Thus, for $\left.\left(z V^{\prime}(z) / V(z)\right)\right|_{z=0}=0$, we have

$$
1+\frac{z V^{\prime}(z)}{V(z)} \prec \wp(z) .
$$

Then, in view of [9], Theorem 3.4.c, we have $V(z) \prec \wp(z)$.

Next, the results show the sufficient and necessary conditions for the sandwich behavior of the functional $\left(\frac{{ }_{\kappa}^{\mathcal{C}} \Omega_{a, b}^{p, q} \varphi(z)}{z^{\rho}}\right)^{v}$.

Theorem 3. Let the following assumptions hold

$$
\left(\frac{{ }_{\kappa}^{\mathcal{C}} \Omega_{a, b}^{p, q} \varphi(z)}{z^{\rho}}\right)^{v}\left(v \frac{z\left({ }_{\kappa}^{\mathcal{C}} \Omega_{a, b}^{p, q} \varphi(z)\right)^{\prime}}{{ }_{\mathcal{K}}^{\mathcal{C}} \Omega_{a, b}^{p, q} \varphi(z)}+(1-v \rho)\right) \prec \wp_{2}(z)+z \wp_{2}^{\prime}(z),
$$

where $\wp_{2}(0)=1$ and convex in $\sqcup$. Moreover, let $\left(\frac{{ }_{\kappa}^{\mathcal{C}} \Omega_{a, b}^{p, q} \varphi(z)}{z^{\rho}}\right)^{v}$ be univalent in $\sqcup$ such that $\left(\frac{{ }_{K}^{\mathcal{C}} \Omega_{a, b}^{p, q} \varphi(z)}{z^{\rho}}\right)^{v} \in \mathbb{O}\left[\wp_{1}(0), 1\right] \cap \mathbb{Q}$, where $\mathbb{Q}$ represents the set of all injection analytic functions $f$ with $\lim _{z \in \partial \sqcup} f \neq \infty$ and

$$
\wp_{1}(z)+z \wp_{1}^{\prime}(z) \prec\left(\frac{{ }_{\kappa}^{\mathcal{C}} \Omega_{a, b}^{p, q} \varphi(z)}{z^{\rho}}\right)^{v}\left(v \frac{z\left({ }_{\kappa}^{\mathcal{C}} \Omega_{a, b}^{p, q} \varphi(z)\right)^{\prime}}{{ }_{\mathcal{K}}^{\mathcal{C}} \Omega_{a, b}^{p, q} \varphi(z)}+(1-v \rho)\right) .
$$

Then,

$$
\wp_{1}(z) \prec\left(\frac{{ }_{\kappa}^{\mathcal{C}} \Omega_{a, b}^{p, q} \varphi(z)}{z^{\rho}}\right)^{v} \prec \wp_{2}(z)
$$

and $\wp_{1}(z)$ is the best sub-dominant and $\wp_{2}$ is the best dominant.

Proof. Since,

$$
\left(\frac{{ }_{\kappa}^{\mathcal{C}} \Omega_{a, b}^{p, q} \varphi(z)}{z^{\rho}}\right)^{v}+z\left(\left(\frac{{ }_{K}^{\mathcal{C}} \Omega_{a, b}^{p, q} \varphi(z)}{z^{\rho}}\right)^{v}\right)^{\prime}=\left(\frac{{ }_{\kappa}^{\mathcal{C}} \Omega_{a, b}^{p, q} \varphi(z)}{z^{\rho}}\right)^{v}\left(v \frac{z\left({ }_{\kappa}^{\mathcal{C}} \Omega_{a, b}^{p, q} \varphi(z)\right)^{\prime}}{{ }_{\mathcal{K}} \Omega_{a, b}^{p, q} \varphi(z)}+(1-v \rho)\right),
$$


then we have the following double inequality

$$
\begin{aligned}
\wp_{1}(z)+z \wp_{1}^{\prime}(z) & \prec\left(\frac{{ }_{\kappa}^{\mathcal{C}} \Omega_{a, b}^{p, q} \varphi(z)}{z^{\rho}}\right)^{v}+z\left(\left(\frac{{ }_{\kappa}^{\mathcal{C}} \Omega_{a, b}^{p, q} \varphi(z)}{z^{\rho}}\right)^{v}\right)^{\prime} \\
& \prec \wp_{2}(z)+z \wp_{2}^{\prime}(z) .
\end{aligned}
$$

Accordingly, by Lemma 1d,e, we have the desired assertion.

Theorem 4. Let $\wp$ be a univalent convex function in $\sqcup$ such that $\wp(0)=1$ and

$$
{ }_{\kappa}^{\mathcal{C}} \Omega_{a, b}^{p, q} \varphi(z) \prec \wp(z), \quad{ }_{\kappa}^{\mathcal{C}} \Xi_{a, b}^{p, q} \varphi(z) \prec \wp(z) .
$$

Then,

$$
{ }_{\kappa}^{\alpha} \partial_{a, b}^{p, q} \varphi(z)=\alpha{ }_{\kappa}^{\mathcal{C}} \Omega_{a, b}^{p, q} \varphi(z)+(1-\alpha){ }_{\kappa}^{\mathcal{C}} \Xi_{a, b}^{p, q} \varphi(z) \prec \wp(z), \quad \alpha \in[0,1] .
$$

Proof. By a direct application of Lemma 1f, we obtain the result.

Special Case

In the next presentation, we focus on a special case, when

$$
\begin{gathered}
\wp(z)=\frac{1+\theta_{1} z}{1+\theta_{2} z}, \\
\left(-1 \leq \theta_{2}<\theta_{1} \leq 1\right) .
\end{gathered}
$$

Theorem 5. Let $\varphi \in \underset{\kappa}{v} \top_{a, b}^{p, q}\left(\frac{1+\theta_{1} z}{1+\theta_{2} z}\right)$. Then,

(i)

$$
\varphi(z)=\left(z^{\rho}\left(\frac{1+\theta_{1} \vartheta(z)}{1+\theta_{2} \vartheta(z)}\right)^{1 / v}\right) *\left(z^{\rho}+\sum_{n=\rho+1}^{\infty}\left(\frac{(t)_{\mathcal{K}} \Gamma(t) \Sigma_{a, s+t}^{-p}\left[q z^{a}\right]}{(\tau)_{\kappa} \Gamma(\tau) \Sigma_{a, s+\tau}^{-p}\left[q z^{a}\right]}\right) z^{n}\right) .
$$

(ii) Moreover, the function $\varphi$ satisfies

$$
\left(1+\theta_{2} e^{i \theta}\right)^{\frac{1}{v}}\left(z^{\rho}+\sum_{n=\rho+1}^{\infty}\left(\frac{(\tau)_{\mathcal{K}} \Gamma(\tau) \Sigma_{a, s+\tau}^{-p}\left[q z^{a}\right]}{(t)_{\mathcal{K}} \Gamma(t) \Sigma_{a, s+t}^{-p}\left[q z^{a}\right]}\right) z^{n}\right) * \varphi(z)-z^{\rho}\left(1+\theta_{1} e^{i \theta}\right)^{\frac{1}{v}} \neq 0 .
$$

(iii) ${ }_{\kappa}^{v} \top_{a, b}^{p, q}\left(\frac{1+\theta_{1} z}{1+\theta_{2} z}\right) \subseteq{ }_{\kappa}^{v} \top_{a, b}^{p, q}\left(\frac{1+\theta_{3} z}{1+\theta_{4} z}\right)$, where $-1 \leq \theta_{4} \leq \theta_{2}<\theta_{1} \leq \theta_{3} \leq 1$.

$$
\left(-1 \leq \theta_{2}<\theta_{1} \leq 1, z \in \sqcup, \vartheta(0)=0,|\vartheta(z)|<1, \theta \in(0,2 \pi)\right)
$$

Proof. Since $\varphi \in{ }_{\kappa}^{v} T_{a, b}^{p, q}\left(\frac{1+\theta_{1} z}{1+\theta_{2} z}\right)$, then there occurs an analytic function $\vartheta: \sqcup \rightarrow \sqcup$ such that

$$
\left(\frac{{ }_{\kappa}^{\mathcal{C}} \Omega_{a, b}^{p, q} \varphi(z)}{z^{\rho}}\right)^{v}=\left(\frac{1+\theta_{1} \vartheta(z)}{1+\theta_{2} \vartheta(z)}\right) .
$$

A computation implies that

$$
{ }_{\kappa}^{\mathcal{C}} \Omega_{a, b}^{p, q} \varphi(z)=z^{\rho}\left(\frac{1+\theta_{1} \vartheta(z)}{1+\theta_{2} \vartheta(z)}\right)^{1 / v} .
$$


By Proposition 1, we have

$$
\begin{aligned}
\varphi(z) & =\varphi(z) *\left(z^{\rho}+\sum_{n=\rho+1}^{\infty}\left(\frac{(\tau)_{\mathcal{K}} \Gamma(\tau) \Sigma_{a, s+\tau}^{-p}\left[q z^{a}\right]}{(t)_{\mathcal{K}} \Gamma(t) \Sigma_{a, s+t}^{-p}\left[q z^{a}\right]}\right) z^{n}\right) \\
& *\left(z^{\rho}+\sum_{n=\rho+1}^{\infty}\left(\frac{(t)_{\mathcal{K}} \Gamma(t) \Sigma_{a, s+t}^{-p}\left[q z^{a}\right]}{(\tau)_{\mathcal{K}} \Gamma(\tau) \Sigma_{a, s+\tau}^{-p}\left[q z^{a}\right]}\right) z^{n}\right) \\
& =\left(z^{\rho}+\sum_{n=\rho+1}^{\infty}\left(\frac{(\tau)_{\mathcal{K}} \Gamma(\tau) \Sigma_{a, s+\tau}^{-p}\left[q z^{a}\right]}{(t)_{\mathcal{K}} \Gamma(t) \Sigma_{a, s+t}^{-p}\left[q z^{a}\right]}\right) \varphi_{n} z^{n}\right) \\
& *\left(z^{\rho}+\sum_{n=\rho+1}^{\infty}\left(\frac{(t)_{\mathcal{K}} \Gamma(t) \Sigma_{a, s+t}^{-p}\left[q z^{a}\right]}{(\tau)_{\mathcal{K}} \Gamma(\tau) \Sigma_{a, s+\tau}^{-p}\left[q z^{a}\right]}\right) z^{n}\right) \\
& =\left({ }_{\kappa}^{\mathcal{C}} \Omega_{a, b}^{p, q} \varphi(z)\right) *\left(z^{\rho}+\sum_{n=\rho+1}^{\infty}\left(\frac{(t)_{\mathcal{K}} \Gamma(t) \Sigma_{a, s+t}^{-p}\left[q z^{a}\right]}{(\tau)_{\mathcal{K}} \Gamma(\tau) \Sigma_{a, s+\tau}^{-p}\left[q z^{a}\right]}\right) z^{n}\right) \\
& =\left(z^{\rho}\left(\frac{1+\theta_{1} \vartheta(z)}{1+\theta_{2} \vartheta(z)}\right)^{1 / v}\right) *\left(z^{\rho}+\sum_{n=\rho+1}^{\infty}\left(\frac{(t)_{\mathcal{K}} \Gamma(t) \Sigma_{a, s+t}^{-p}\left[q z^{a}\right]}{(\tau)_{\kappa} \Gamma(\tau) \Sigma_{a, s+\tau}^{-p}\left[q z^{a}\right]}\right) z^{n}\right),
\end{aligned}
$$

which completes the proof.

The assumption of second part implies that

$$
\left(\frac{{ }_{\kappa}^{\mathcal{C}} \Omega_{a, b}^{p, q} \varphi(z)}{z^{\rho}}\right)^{v} \prec \frac{1+\theta_{1} z}{1+\theta_{2} z^{\prime}}
$$

which means that

$$
\left(\frac{{ }_{\kappa}^{\mathcal{C}} \Omega_{a, b}^{p, q} \varphi(z)}{z^{\rho}}\right)^{v} \neq \frac{1+\theta_{1} e^{i \theta}}{1+\theta_{2} e^{i \theta}} .
$$

By rearranging the above relation, we have

$$
\left(\left(1+\theta_{2} e^{i \theta}\right)^{\frac{1}{v}}\left({ }_{\kappa}^{C} \Omega_{a, b}^{p, q} \varphi(z)\right)-z^{\rho}\left(1+\theta_{1} e^{i \theta}\right)^{\frac{1}{v}}\right) \neq 0 .
$$

Proposition 1 yields

$$
\left(1+\theta_{2} e^{i \theta}\right)^{\frac{1}{v}}\left(z^{\rho}+\sum_{n=\rho+1}^{\infty}\left(\frac{(\tau)_{\mathcal{K}} \Gamma(\tau) \Sigma_{a, s+\tau}^{-p}\left[q z^{a}\right]}{(t)_{\mathcal{K}} \Gamma(t) \Sigma_{a, s+t}^{-p}\left[q z^{a}\right]}\right) z^{n}\right) * \varphi(z)-z^{\rho}\left(1+\theta_{1} e^{i \theta}\right)^{\frac{1}{v}} \neq 0 .
$$

We proceed to show the last assertion. In view of the assumption $\varphi \in{ }_{\kappa}^{v} \top_{a, b}^{p, q}\left(\frac{1+\theta_{1} z}{1+\theta_{2} z}\right)$, we obtain

$$
\left(\frac{{ }^{\mathcal{C}} \Omega_{a, b}^{p, q} \varphi(z)}{z^{\rho}}\right)^{v} \prec \frac{1+\theta_{1} z}{1+\theta_{2} z}
$$

However,

$$
\begin{gathered}
\frac{1+\theta_{1} z}{1+\theta_{2} z} \prec \frac{1+\theta_{3} z}{1+\theta_{4} z} \\
\left(-1 \leq \theta_{4} \leq \theta_{2}<\theta_{1} \leq \theta_{3} \leq 1\right) .
\end{gathered}
$$

Thus, we obtain

$$
\varphi \in \underset{\kappa}{v} \top_{a, b}^{p, q}\left(\frac{1+\theta_{3} z}{1+\theta_{4} z}\right)
$$




\section{Conclusions}

A fractional differential operator in the open unit disk is presented for multivalent analytic functions. We formulated the modified operator in two sub-classes of analytic functions and studied the geometric behavior. Differential inequalities are presented using the theory of subordination and superordination. Our main result is given in Theorem 3, where the conditions of the sandwich inequality are presented. Moreover, Theorem 4 showed the convex combination of the differential operator and its corresponding integral, which are dominated by $\wp(z)$, which is dominated by the same analytic function $\wp(z)$.

Author Contributions: Conceptualization, R.W.I. and N.M.A.; methodology, R.W.I. and N.M.A.; formal analysis, R.W.I.; investigation, R.W.I. and N.M.A.; funding acquisition, N.M.A. All authors have read and agreed to the published version of the manuscript.

Funding: This research received no external funding.

Institutional Review Board Statement: Not applicable.

Informed Consent Statement: Not applicable.

Data Availability Statement: No new data were created or analyzed in this study. Data sharing is not applicable to this article.

Acknowledgments: The authors would like to express their full thanks to the respected editorial office.

Conflicts of Interest: The authors declare no conflict of interest.

\section{References}

1. Salagean, G.S. Subclasses of Univalent Functions, Complex Analysis-Fifth Romanian-Finnish Seminar, Part 1, Lecture Notes in Mathematics, Volume 1013; Springer: Berlin, Germany, 1983; pp. 362-372.

2. Al-Oboudi, F.M. On univalent functions defined by a generalized Salagean operator. Int. J. Math. Math. Sci. 2004, 27, 1429-1436. [CrossRef]

3. Ibrahim, R.W.; Darus, M. Univalent functions formulated by the Salagean-difference operator. Int. J. Anal. Appl. 2019, 17, 652-658.

4. Ibrahim, R.W.; Jahangiri, J. Conformable differential operator generalizes the Briot-Bouquet differential equation in a complex domain. AIMS Math. 2019, 4, 1582. [CrossRef]

5. Ibrahim, R.W.; Darus, M. New symmetric differential and integral operators defined in the complex domain. Symmetry 2019, 11, 906. [CrossRef]

6. Srivastava, H.M.; Saigo, M.; Owa, S. A class of distortion theorems involving certain operators of fractional calculus. J. Math. Anal. Appl. 1988, 131, 412-420. [CrossRef]

7. Ibrahim, R.W. On generalized Srivastava-Owa fractional operators in the unit disk. Adv. Differ. Equ. 2011, 2011, 55. [CrossRef]

8. Abdulnaby, Z.E.; Rabha, W.I. On a subclass of analytic functions of fractal power with negative coefficients. Bull. Transilv. Univ. Brasov. Math. Inform. Physics. Ser. III 2020, 13, 387-398. [CrossRef]

9. Miller, S.S.; Mocanu, P.T. Differential Subordinations: Theory and Applications; CRC Press: Boca Raton, FL, USA, 2000.

10. Ma, W.C.; Minda, D. A unified treatment of some special classes of univalent functions. In Proceedings of the Conference on Complex Analysis, Tianjin, China, 19-23 June 1992; International Press: Cambridge, MA, USA, 1994; pp. 157-169.

11. Ali, R.M.; Lee, S.K.; Ravichandran, V.; Supramaniam, S. Coefficient estimates for bi-univalent Ma-Minda starlike and convex functions. Appl. Math. Lett. 2012, 25, 344-351. [CrossRef]

12. Seoudy, T.M.; Aouf, M.K. Coefficient estimates of new classes of q-starlike and q-convex functions of complex order. J. Math. Inequal 2016, 10, 135-145. [CrossRef]

13. Masih, V.S.; Kanas, S. Subclasses of starlike and convex functions associated with the limacon domain. Symmetry 2020, $12,942$. [CrossRef]

14. Ebadian, A.; Mohammed, N.H.; Adegani, E.A.; Bulboaca, T. New results for some generalizations of starlike and convex functions. J. Funct. Spaces 2020, 2020, 7428648. [CrossRef]

15. Karthikeyan, K.R.; Murugusundaramoorthy, G.; Cho, N.E. Some inequalities on Bazilevic class of functions involving quasisubordination. AIMS Math. 2021, 6, 7111-7124.

[CrossRef]

16. Choi, J.; Datta, S.K.; Biswas, N. Growth Analysis of Meromorphic Solutions of Linear Difference Equations with Entire or Meromorphic Coefficients of Finite $\varphi$-Order. Symmetry 2021, 13, 267. [CrossRef]

17. Srivastava, H.M. Some families of Mittag-Leffler type functions and associated operators of fractional calculus (Survey). TWMS J. Pure Appl. Math. 2016, 7, 123-145.

18. Shukla, A.K.; Prajapati, J.C. On a generalization of Mittag-Leffler function and its properties. J. Math. Anal. Appl. 2007, 336, 797-811. [CrossRef] 
19. Haubold, H.J.; Arak M.M.; Ram, K.S. Mittag-Leffler functions and their applications. J. App. Math. 2011, 2011, 298628. [CrossRef]

20. Prabhakar, T.R. A singular integral equation with a generalized Mittag-Leffler function in the kernel. Yokohama Math. J. 1971, 19, 7-15.

21. Garra, R.; Gorenflo, R.; Polito, F.; Tomovski, Ž. Hilfer-Prabhakar derivatives and some applications. Appl. Math. Comp. 2014, 242, 576-589. [CrossRef]

22. Kilbas, A.A.; Megumi S.; Ram, K.S. Generalized Mittag-Leffler function and generalized fractional calculus operators. Int. Trans. Spec. Func. 2004, 15, 31-49. [CrossRef]

23. Shanmugam, T.N.; Sivasubramanian, S.; Srivastava, H.M. Differential sandwich theorems for certain subclasses of analytic functions involving multiplier transformations. Int. Trans. Spec. Func. 2006, 17, 889-899. [CrossRef]

24. Miller, S.S.; Mocanu, P.T. Subordinants of differential superordinations. Comp. Vari. 2003, 48, 815-826. [CrossRef]

25. Guo, D.; Ming-Sheng, L. On certain subclass of Bazilevic functions. J. Inequal. Pure Appl. Math 2007, 8, 1-11.

26. Rogosinski, W. On the coefficients of subordinate functions. Proc. Lond. Math. Soc. 1945, 2, 48-82. [CrossRef] 